\title{
Whether ideal free or not, predatory mites distribute so as to maximize reproduction
}

\author{
Tessa van der Hammen • Marta Montserrat • \\ Maurice W. Sabelis • André M. de Roos • \\ Arne Janssen
}

Received: 9 March 2011/Accepted: 17 October 2011/Published online: 12 November 2011

(C) The Author(s) 2011. This article is published with open access at Springerlink.com

\begin{abstract}
Ideal free distribution (IFD) models predict that animals distribute themselves such that no individual can increase its fitness by moving to another patch. Many empirical tests assume that the interference among animals is independent of density and do not quantify the effects of density on fitness traits. Using two species of predatory mites, we measured oviposition as a function of conspecific density. Subsequently, we used these functions to calculate expected distributions on two connected patches. We performed an experimental test of the distributions of mites on two such connected patches, among which one had a food accessibility rate that was twice as high as on the other. For one of the two species, Iphiseius degenerans, the distribution matched the expected distribution. The distribution also coincided with the ratio of food accessibility. The other species, Neoseiulus cucumeris, distributed itself differently than expected. However, the oviposition rates of both species did not differ significantly from the expected
\end{abstract}

Communicated by Andreas Prinzing.

T. van der Hammen - M. Montserrat - M. W. Sabelis .

A. M. de Roos - A. Janssen

IBED, Section Population Biology, University of Amsterdam,

Amsterdam, The Netherlands

Present Address:

T. van der Hammen ( $\square)$

IMARES Wageningen UR, Institute for Marine Resources and

Ecosystem Studies, PO Box 68, 1970 AB IJmuiden,

The Netherlands

e-mail: tessa.vanderhammen@wur.nl

Present Address:

M. Montserrat

Instituto de Hortofruticultura Subtropical y Mediterránea "La

Mayora” (IHSM-UMA-CSIC), Algarrobo-Costa,

29750 Málaga, Spain oviposition rates based on experiments on single patches. This suggests that the oviposition rate of $N$. cucumeris was not negatively affected by the observed distribution, despite the fact that $N$. cucumeris did not match the predicted distributions. Thus, the distribution of one mite species, I. degenerans, was in agreement with IFD theory, whereas for the other mite species, $N$. cucumeris, unknown factors may have influenced the distribution of the mites. We conclude that density-dependent fitness traits provide essential information for explaining animal distributions.

Keywords Competition - Game theory · Habitat selection · Interference · Phytoseiids

\section{Introduction}

Resources are often patchily distributed, and patches usually differ in quality because of varying amounts of resources and numbers of competitors. Foraging theory predicts that animals distribute themselves over patches in such a way that they maximize their fitness (Charnov 1976; Milinski and Parker 1991; Pulliam and Caraco 1984). A well-known and simple theory that predicts the optimal distribution of animals in a competitive environment is that of the ideal free distribution (IFD, Fretwell and Lucas 1969; Kacelnik et al. 1992; Milinski and Parker 1991; Parker 1978; Tregenza 1995). This theory assumes that all animals are "ideal", i.e. they have perfect information about the quality of the patches, and "free", meaning that they are able to move among patches without incurring a cost. Therefore, the animals are expected to distribute themselves such that no individual can increase its fitness by moving to another patch (Fretwell and Lucas 1969; Milinski and Parker 1991; Parker 1978; Tregenza 1995). 
There are two variants of the IFD model; the "immediate consumption" model (also called the "continuous input" model) and the "interference" model. In the first model, resource items arrive at a constant rate, and all items are consumed instantaneously. In the interference model, the gain rate is assumed to be reduced by the presence of others due to interference, such as fighting, kleptoparasitism, or disturbance of the prey (Parker and Sutherland 1986; Sutherland and Parker 1992; Tregenza 1994, 1995). Empirical studies usually test the immediate consumption model with two patches that differ in food availability (Milinski 1984; Milinski and Parker 1991; Tregenza 1995). They assume that there is no interference, and compare the fraction of individuals in a patch with the fraction of food in that patch. Although many studies show that the animals distribute themselves in the same ratio as the food input on these patches (Milinski 1984; Milinski and Parker 1991; Tregenza 1995), distributions also often deviate from food supply rates, usually with more competitors than expected on the patches of lower quality ("undermatching," Abrahams 1986; Earn and Johnstone 1997; Fretwell 1972; Kennedy and Gray 1993; Milinski and Parker 1991; Sutherland 1983). The deviations are generally explained by the violation of either assumption (that the animals are ideal or free) or both (Abrahams 1986; Fretwell 1972; Holmgren 1995; Hugie and Grand 1998). Another explanation of the deviations from the food input rates is that there is interference such as fighting, kleptoparasitism, or indirect effects through the prey (Tregenza 1994; Moody and Houston 1995; Moody and Ruxton 1996, Van der Meer and Ens 1997). In such cases, individuals are expected to avoid patches with high densities of competitors, so the distribution will undermatch the ratio of food supply. Alternatively, if competition for food is weak, some food items may remain uneaten, which may also lead to deviations from the expected distribution. In addition, interference could also increase with competitor densities (Moody and Houston 1995; Moody and Ruxton 1996). With interference, a distribution is not expected to match the ratio of food input, but it may nevertheless be an IFD with respect to the fitness of the individuals (Flaxman and deRoos 2007; Sutherland and Parker 1992; Tregenza 1994). The fitness of each competitor may be equal, but this does not necessarily coincide with equal ratios of food supply per individual and patch. Testing whether animals distribute themselves such that no individual can increase its fitness by moving to another patch without specifically testing either the immediate consumption model or the interference model would thus involve estimating the relationship between density and fitness traits, and subsequently using this relationship to predict the distribution (Flaxman and deRoos 2007; Haugen et al. 2006; Sutherland and Parker 1992; Tregenza 1994; Tregenza et al. 1996a). In contrast with experimental studies that test the matching of food input only, there are only a few studies that have measured such density-dependent estimates of fitness traits and used them to predict the IFD (Flaxman and deRoos 2007; Haugen et al. 2006; Morris 1989; Tregenza et al. 1996b).

In this paper, we present two empirical tests of the ideal free distribution using two species of predatory mites. To estimate the effects of competition, we used the oviposition rates of the mites, because this rate depends strongly on the availability of food. For each mite species, we conducted two sets of experiments. The first set was performed on single patches, where we tested the effect of density on the oviposition rates of the mites. We varied the accessibility of food as well as the numbers of competitors. We expected the oviposition rate to increase with the level of food accessibility and to decrease with increasing numbers of competitors. We used these data in a regression analysis to estimate the relationship between food accessibility and oviposition rate for each patch separately (Flaxman and deRoos 2007; Sutherland and Parker 1992; Tregenza 1994). In the second empirical test, we connected two patches that differed in food accessibility such that one patch had twice as much access to food as the other. We allowed the mites to distribute themselves between the patches and we analyzed the observed distribution in three ways. First, we tested whether the distribution was random (1:1), and second, whether it matched food accessibility rates (1:2). Third, we used the estimates from the regression analysis from the single patch experiments and calculated an expected distribution based on oviposition rates, which we compared with the observed distribution. In addition, oviposition rates from the single patches were tested against oviposition rates on the connected patches. We expected the oviposition rate of the mites to equal that observed on the single patches with the same ratio of food accessibility and number of competitors.

\section{Materials and methods}

\section{Experimental system}

The predatory mites used in this study were Iphiseius degenerans and Neoseiulus cucumeris. Both species are omnivores and feed on a variety of food sources, such as thrips larvae and pollen of several plant species (Van Rijn and Tanigoshi 1999, Vantornhout 2006). Both species allocate a large fraction of their food intake to reproduction; the daily egg mass produced by a female of I. degenerans females is about $40 \%$ of its full adult body weight (Yao and Chant 1990). Both mites are very mobile, especially $I$. degenerans, which can easily move to any place on the arena within a few seconds. Neoseiulus 
cucumeris is less mobile, but can still quickly move to any place on the arena. In addition, the mites use chemical cues to assess the quality of their environment (Choh et al. 2010); hence, they may react to each other's presence without physical contact. The two predatory mites co-occur in the Mediterranean (De Moraes et al. 2004) and are used in various crops as biological control agents against several thrips species. The size of $I$. degenerans is $0.55 \pm 0.003 \mathrm{~mm}$ in length and $0.36 \pm 0.002 \mathrm{~mm}$ in width (Vantornhout 2006). The size of $N$. cucumeris is similar to $N$. communis, which is $0.43-0.44 \mathrm{~mm}$ in length and $0.24-0.25 \mathrm{~mm}$ in width (Denmark and Edland 2002). These two species are usually cultured on artificial substrates and do well on a diet of pollen (Vantornhout 2006; Van Rijn and Tanigoshi 1999).

\section{Cultures}

Iphiseius degenerans and $N$. cucumeris were cultured in a climate room at $25 \pm 1{ }^{\circ} \mathrm{C}, 16: 8 \mathrm{~h} \mathrm{~L} / \mathrm{D}$, and $60 \pm 5 \% \mathrm{RH}$. Mites were held on plastic arenas $(30 \times 21 \mathrm{~cm})$ placed on top of sponges in water-containing trays. Small threads $(3-5 \mathrm{~cm})$ were added to the arenas and served as oviposition sites. Neoseiulus cucumeris was fed cattail pollen (Typha ssp.) and I. degenerans birch pollen (Betula ssp.), both twice per week. Every week, eggs were collected from the cultures and placed on new plastic arenas to obtain cohorts of mites from a similar age. For more details on the culture methods employed, see Van Rijn and Tanigoshi (1999). Experiments were carried out in a climate room at $25 \pm 1{ }^{\circ} \mathrm{C}, 16 \mathrm{~h}$ light per day, and $60 \pm 5 \% \mathrm{RH}$.

To make the differences among individuals as small as possible, all mites used in the experiments were adult females that were 10-15 days old, corresponding to the age at which food intake and oviposition rates are highest (Van Rijn and Tanigoshi 1999).

\section{Experimental setup}

We developed a setup in which animals competed for access to the food source instead of competing for the food itself. This was implemented by filling small holes $(\varnothing$ $0.5 \mathrm{~mm}, 1 \mathrm{~cm}$ deep) made in plastic arenas $(8 \times 15 \times 1 \mathrm{~cm}$, Fig. 1) with cattail (Typha ssp.) pollen, which both mite species use as food. Because of the small diameter of the hole, only 1-3 I. degenerans or 2-4 N. cucumeris had access to food at any particular time. In this system, access to food was constant during the experiment and the space around and inside the holes was continuously occupied when the density of I. degenerans was 10 or higher or that of $N$. cucumeris was 50 or higher. When a mite left its position at the hole, its place was taken instantly by another mite. Permanent monopolization of the
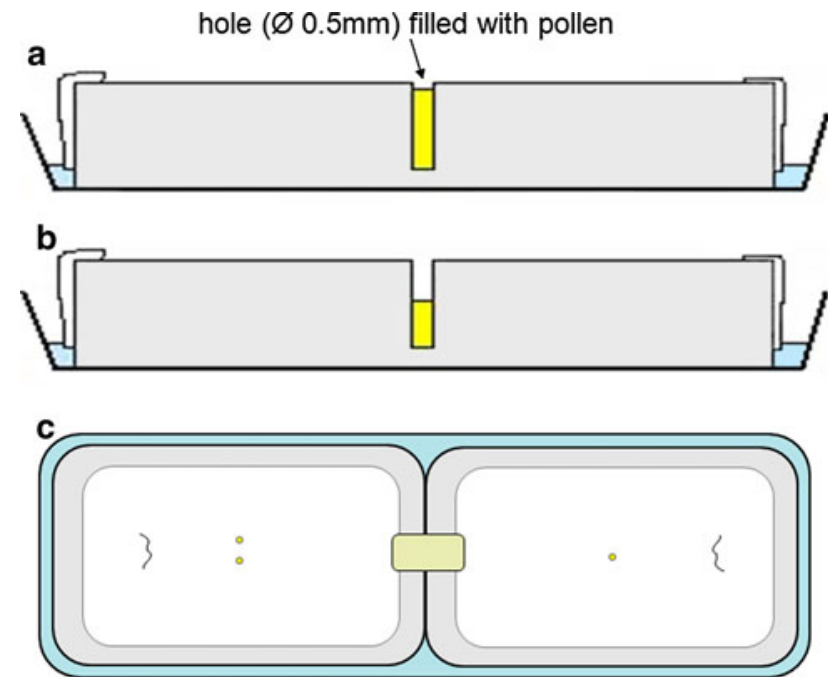

Fig. 1 Experimental setup, consisting of a plastic patch $(8 \times 15 \mathrm{~cm})$ that had either one or two holes $(\varnothing 0.5 \mathrm{~mm})$ filled with pollen $(\mathbf{a})$. When the mites feed on the pollen, the pollen level in the holes goes down, but the hole diameter remains constant (b), resulting in constant accessibility to the pollen. Patches with one or two holes with pollen were connected by a bridge, and a small piece of thread served as an oviposition site on each arena (c)

food by the same individuals did not occur because the mites oviposited elsewhere on the arena, thus making way for other mites. The pollen in the holes went down because of feeding, but because the diameter of the holes remained constant and the mites can easily descend into the holes and ascend from the holes, the rate of food accessibility remained approximately the same and depended on the occupation of the holes by other mites (Fig. 1). Pollen was never depleted during the experiments (i.e. the holes were never empty), and we expected that competition for access to the food would occur only when the mite densities were high enough. This is because the level of competition on the patches with one hole and on those with two holes would be equal with low mite densities because each mite would have access to the pollen and could thus feed at its maximum rate (hence, resource matching is not expected at low densities). Because the holes were only accessible by a few mites at any time, we expected the level of competition to increase rapidly with mite density. Patches of different quality were obtained by making arenas with either one or two holes. The holes were situated in the middle of the arena. In the case of the arenas with two holes, each of them were $0.75 \mathrm{~cm}$ away from the middle (i.e. $1 \frac{1}{2} \mathrm{~cm}$ apart, Fig. 1c). Wet tissue paper on the sides of the arenas served as a source of water and prevented the mites from escaping, and pieces of thread $( \pm 3 \mathrm{~cm})$ were added to the arenas, away from the holes with pollen, to provide ample space for oviposition. Competition for oviposition sites did not occur during the experiment, because the thread provided enough space for all eggs. In addition, both predatory 
mite species prefer to oviposit in clusters (Faraji et al. 2000, 2002b). We will refer to the arenas with one hole as "high competition patches" and to the arenas with two holes as "low competition patches".

Density effects on single patches

To assess the effect of competition on oviposition rates, we placed $1,10,25$, or 50 adult female I. degenerans or 1, 50, or 100 adult female $N$. cucumeris (10-16 replicates per patch, see caption of Fig. 2) on an arena (a patch) with one or two holes filled with pollen (Fig. 1). We used higher numbers of $N$. cucumeris than of $I$. degenerans because the former are smaller, causing competition around the holes with pollen to be weaker. For each density, we added a treatment with sufficient pollen, but spread over the arena instead of offered inside a hole, so that there was no competition for access to the food. We will refer to these patches as "controls". The eggs oviposited after $24 \mathrm{~h}$ and after $48 \mathrm{~h}$ were counted under a stereomicroscope, and the average number of eggs between 24 and $48 \mathrm{~h}$ was used as an estimate for the effect of competition. Eggs laid on the
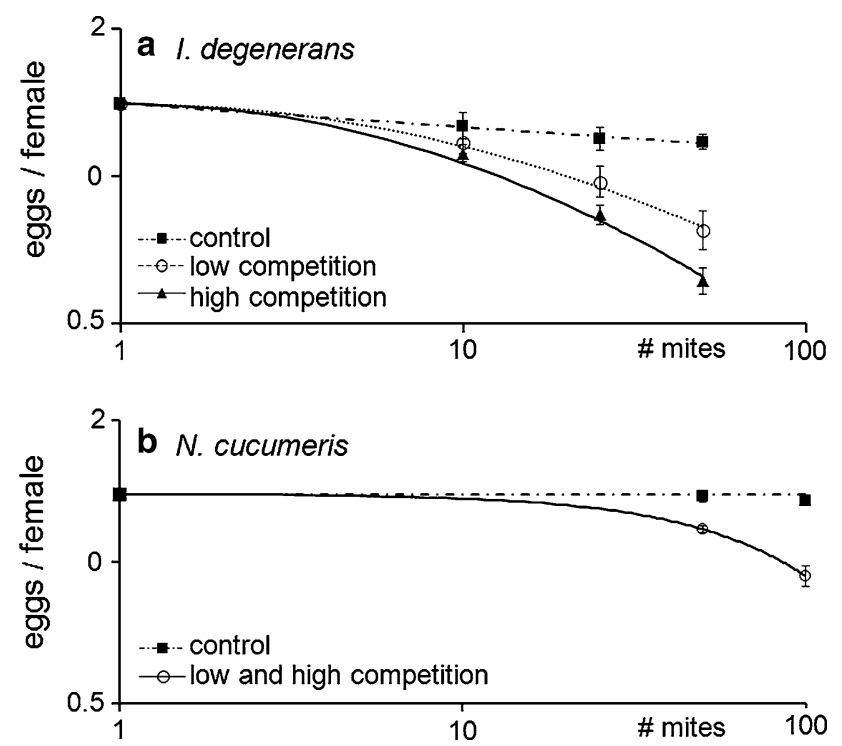

Fig. 2 Log-log plot of the oviposition rates on control patches with ample food (pollen, no competition), patches with one hole with pollen (high competition), and patches with two holes with pollen (low competition) at different mite densities. a I. degenerans, b $N$. cucumeris (high and low competition combined). The lines are the fitted regression lines. See the text for details. Sample sizes: $I$. degenerans 1 mite: $N=14$ (control), $N=13$ (low competition), $N=14$ (high competition); 10 mites: $N=14$ (control), $N=13$ (low competition), $N=15$ (high competition); 25 mites: $N=14$ (control), $N=12$ (low competition), $N=13$ (high competition); 50 mites: $N=14$ (control), $N=12$ (low competition), $N=13$ (high competition). $N$. cucumeris 1 mite: $N=15$ (control), $N=30$ (combined, 15 low competition, 15 high competition); 50 mites: $N=10$ (control), $N=20$ (combined, 10 high competition, 10 low competition); 100 mites: $N=10$ (control), $N=16$ (high competition) first day were not included in the analysis because the first eggs had partly developed when the mites were still in the culture, which could result in an overestimation of oviposition rates.

Regression analysis of single patches

The fitness in patch $i, W_{i}$, is calculated as:

$W_{i}=Q_{i} / N_{i}^{-m}$

(Sutherland and Parker 1992; Tregenza 1995), where $Q_{i}$ is the profitability of patch $i, N_{i}$ is the number of competitors on patch $i$, and $m$ is the coefficient of interference, which expresses the degree to which consumers in the same patch negatively influence each other's fitness (Hassell and Varley 1969; Sutherland and Parker 1992; Tregenza 1995).

We calculated the maximum oviposition, $Q$ (which equals the fitness, $W$, at the density of one mite, Eq. 1), as the oviposition rate at the density of one mite per patch. We tested for differences between the control and the high and low competition patches (GLM with Poisson errors), and because no differences were found, a single estimate of $Q$ was calculated for each species. Equation 1 can be written as

$\log W=-m \log N+\log Q$.

If this function is linear in $\log N, m$ equals the slope of the regression function (Hassell and Varley 1969; Milinski and Parker 1991; Sutherland and Parker 1985; Tregenza 1995; Tregenza et al. 1996a, b). However, the relationship between $\log W$ and $\log N$ is not linear when interference increases with density. Therefore, we also fitted two functions in which $m$ depended on the density: $m(N)=$ $a \log (N)+b$ and $m(N)=a N+b$, and chose the function with the best fit by starting with a full model and using model simplification to delete nonsignificant terms. Subsequently, the best fit between models was assessed by selecting the model with the lowest Akaike information criterion (AIC). Equation 2 thus became

$\log W=-m(N) \log N+\log Q$.

Distribution of mites

Time series

To test how the mites distributed themselves, a patch with high competition (one hole) was connected to a patch with low competition (two holes) using a small plastic bridge (Fig. 1c). On this bridge, we placed either 75 I. degenerans $(N=10)$ or $150 N$. cucumeris $(N=10)$, and the mites could subsequently move freely between the two patches for $48 \mathrm{~h}$. We counted the number of mites on pictures that were taken of each patch and the bridge at $0,2,4,6,24,26$, 
28,30 , and $48 \mathrm{~h}$ after the start of the experiment using a digital camera, resulting in a time series of the mite distributions. Counts of the mites from the pictures after $48 \mathrm{~h}$ were closely correlated with destructive counts peformed under a stereomicroscope (I. degenerans: $R=0.97$, $d f=18, p<0.001, N$. cucumeris: $R=0.78, d f=18$, $p<0.001$, Pearson's product-moment correlation). Experiments lasted for $48 \mathrm{~h}$, because eggs started to hatch after this time and the presence of larvae could have affected the distribution, as they are a source of food for the cannibalistic adult female mites (Montserrat et al. 2006).

For the analysis of the time series, we used generalized linear mixed effect models with a quasi-binomial error distribution (function lmer of the library lme4 in $R, \mathrm{R}$ Development Core Team (2008)), with time as fixed effect and replicate as a random effect to correct for pseudoreplication due to repeated measurements.

In two replicates of each treatment, we videotaped the bridge that connected the patches with a time-lapse video recorder (Sony STV-S3050P with a Kappa CF 11/3 camera mounted on a binocular microscope) to assess whether the mites did indeed cross frequently. We analyzed the tape for the first half hour of every $2 \mathrm{~h}$ of the experiment by counting the mites that crossed the bridge. From these data, we estimated the frequency of movement of mites between the two patches during the experiment.

\section{Distribution after $48 \mathrm{~h}$}

After $48 \mathrm{~h}$, we disconnected the patches and subsequently counted the mites and the eggs on each patch using a stereomicroscope. We did not count eggs at other time steps because this may have disturbed the mites and thus could have affected their distribution.

We tested the distribution of the mites at the end of the experiments (after $48 \mathrm{~h}$ ) against a random distribution (1:1) and against the ratio of food accessibility on the two arenas (2:1). We tested each replicate separately with $G$ tests for goodness of fit and we assessed the homogeneity of the replicates (Sokal and Rohlf 1995). In addition, we performed one-sample $t$ tests on the fraction of mites on the patches with low competition to test for overall deviations from random distributions and from food accessibility.

Subsequently, we used Eq. 1 to calculate the expected fraction of mites on each patch if they were to maximize their oviposition rate. If the mites distribute themselves according to an IFD, the oviposition rates of the individuals on each patch should be equal, and thus: $Q / N_{1}^{m_{1}}=Q / N_{2}^{m_{2}}$. Because $N_{2}=$ (total mites $-N_{1}$ ) and the interference coefficients $m_{1}$ and $m_{2}$ can be derived from the regression equations as described above, $N_{1}$ and $N_{2}$ can be estimated through iteration. As above, we compared the observed distribution with the expected distribution for each replicate separately using $G$ tests for goodness of fit, and we performed one-sample $t$ tests on the fraction of mites on the patches with low competition to test for overall differences from the expected distribution.

Although we define a patch as one half of the arena separated by a bridge from another patch, the mites may perceive the arena differently. For example, they may perceive each hole as a patch. In this case, one would expect an equal number of mites near each hole, i.e. $1 / 3$ of the mites on the patch with one hole and $2 / 3$ on the patch with two holes. This distribution is the same as that expected under a distribution equal to the ratio of food accessibility. Alternatively, the mites might also perceive the entire arena as a single patch, in which case they would be expected to distribute randomly over the arena. Hence, whichever way the mites perceive the patches, our tests serve to verify their distribution.

\section{Expected oviposition rates}

Equation 1 was used to estimate expected oviposition rates between 24 and $48 \mathrm{~h}$, but we could not compare these estimates with observed oviposition rates because we did not count the eggs at $24 \mathrm{~h}$ in the distribution experiment, as it could have altered the distribution of the mites. Therefore, we compared oviposition rates in the distribution experiment with the oviposition rates of the entire $48 \mathrm{~h}$ of the single patch experiments. This means that eggs were included that may have partly developed when the mites were still in the culture, which may have reduced the differences in observed oviposition rates among treatments. We tested whether the patches with similar densities had the same oviposition rates (i.e. 25 and 50 mites on high and low competition patches, respectively, for I. degenerans, and 50 and 100 mites on patches with high and low competition, respectively, for $N$. cucumeris, one-way ANOVA).

\section{Results}

Regression analysis

\section{Iphiseius degenerans}

The oviposition rate did not differ among patch types at the density of one mite per patch (2.20 eggs per mite, generalized linear model with Poisson errors: $\chi^{2}=0.061$, $d f=1, p=0.98)$. On patches with high and low competition, the minimal adequate regression models of oviposition as a function of mite density were quadratic (Fig. 2a), whereas for the control with ample pollen, a linear model gave the best fit (Fig. 2a). The best-fitting 
model was $\log (W)=-0.28(\log (N))^{2}+0.34\left(R^{2}=0.70\right.$, $p<0.0001)$ and $\log (W)=-0.20(\log (N))^{2}+0.34\left(R^{2}=\right.$ $0.27, p<0.001)$ for the treatments with high and low competition, respectively, and $\log (W)=-0.11 \log (N)+$ $0.34\left(R^{2}=0.51, p<0.0001\right)$ for the treatment with ample pollen. This results in estimates for $m$ of $0.28 \log (N)(95 \%$ conf. int. $0.25 \log (N)$ to $0.31 \log (N))$ and $0.20 \log (N)(95 \%$ conf. int. $0.16 \log (N)-0.24 \log (N))$ for the treatments with high and low competition, and $m=0.11$ (95\% conf. int. 0.07-0.15) for the control treatment. This latter result indicates that oviposition decreased with density even when there was ample food. This means that there was interference even when there was no competition for access to the food.

\section{Neoseiulus cucumeris}

For $N$. cucumeris, the oviposition rate also did not differ among patch types at a density of one mite per patch (GLM with Poisson errors: $\chi^{2}=0.015, d f=1, \quad p=0.90$ ), resulting in an estimated oviposition of 2.15 eggs in $24 \mathrm{~h}$. There was no significant effect of density on oviposition rates on the control patches with ample pollen (Fig. 2b), suggesting that no interference occurred among $\mathrm{N}$. $\mathrm{Cu}$ cumeris when there was ample food. Because of this absence of interference, we expected that the level of competition on the low-competition patches with 100 mites would be equal to that on the high competition patches with 50 mites, because the ratio between mites and the number of holes with pollen was the same in these two treatments. Indeed, the oviposition per mite did not differ significantly between these two treatments (mean (low competition $)=1.53$ eggs $/$ mite, mean $($ high competition $)=1.38$ eggs/mite, $t=1.52, d f=18, p=0.15)$. The data for these two treatments were therefore combined. Consequently, the regression of oviposition as a function of density could be replaced by one of oviposition as a function of the number of mites per hole, resulting in $\log (W)=$ $-0.0022 N \log (N)+0.33\left(R^{2}=0.31, p<0.001\right.$, Fig. 2b). This results in an estimate for $m$ of $0.0022 N$ (conf. int. $0.0019 N-0.0025 N$, hence it differs significantly from 0 for all values of $N$ ).

Distribution of mites on two connected patches

\section{Iphiseius degenerans}

Movements of the mites Analysis of video recordings showed that $I$. degenerans frequently crossed the bridge (on average $30.4 \pm 8.7$ times per mite in $48 \mathrm{~h}$ ). Throughout the experiment, the fraction of mites on the patch with low competition was significantly higher than that on the patch with high competition (LMER, $\chi^{2}=10.15, d f=1$, $p=0.001$, Fig. 3a). The interaction of patch type with time was also significant $\left(\chi^{2}=52.52, d f=1, p<0.001\right)$, suggesting that the difference between the fractions of mites on the patches increased significantly during the experiment (Fig. 3a).

Distribution of the mites The distribution of I. degenerans after $48 \mathrm{~h}$ deviated significantly from a random distribution in seven out of ten replicates (Fig. 4a), whereas only one replicate deviated from the food accessibility ratio (i.e. $1 / 3$ of the mites on the patch with high competition, Fig. 4a). There was no significant heterogeneity between replicates $\left(G_{\mathrm{H}}=10.27, d f=9, p=0.33\right)$, and, on average, the fraction of mites found on the patch with low competition was 0.65 , which did not deviate significantly from the food accessibility rate of 0.67 (Fig. $4 \mathrm{a}, t=1.02$, $d f=9, p=0.33)$, but did deviate significantly from a random distribution of $0.5(t=7.77, d f=9, p<0.001)$.

Fitness estimates based on the oviposition on single patches (Fig. 2a), with estimates of $m$ combined with Eq. 1, yielded a predicted distribution with a fraction of mites on the patch with low competition of 0.64 . None of the ten replicates deviated significantly from this
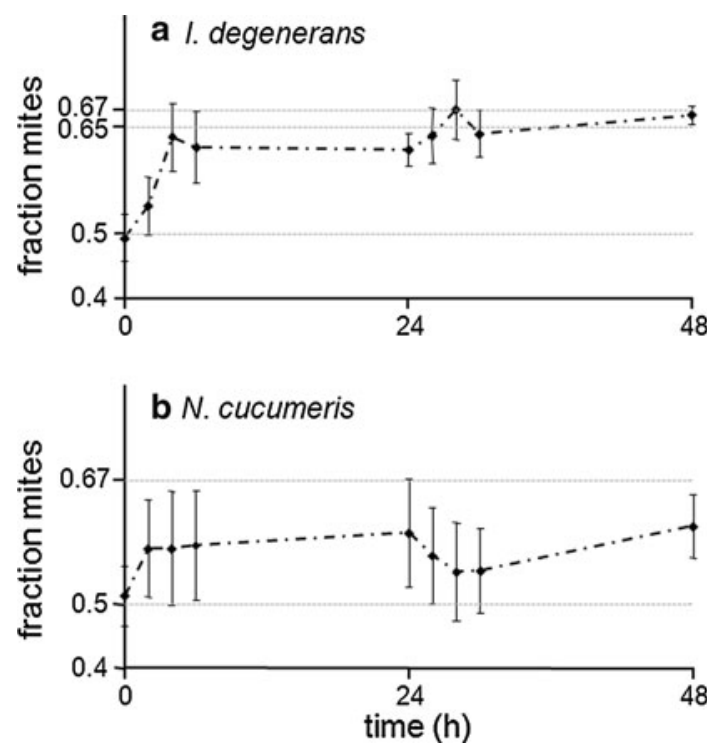

Fig. 3 The distribution of mites over low-competition patches and high-competition patches through time. Shown are the average $( \pm \mathrm{SE})$ fractions of mites on the low-competition patches. Data were collected from pictures taken of the arenas at $0,2,4,6,24,26,28$, 30 , and $48 \mathrm{~h}$ after the introduction of the mites onto the arena. The horizontal lines are the three expected distributions: for I. degenerans (a), these are the random distribution (0.5), the food accessibility rate (0.67), and the distribution based on fitness (0.65, see text). For $N$. cucumeris (b), the expected distributions are the random distribution $(0.5)$, and the distribution based on the food accessibility rate or on oviposition rate $(0.67)$ 


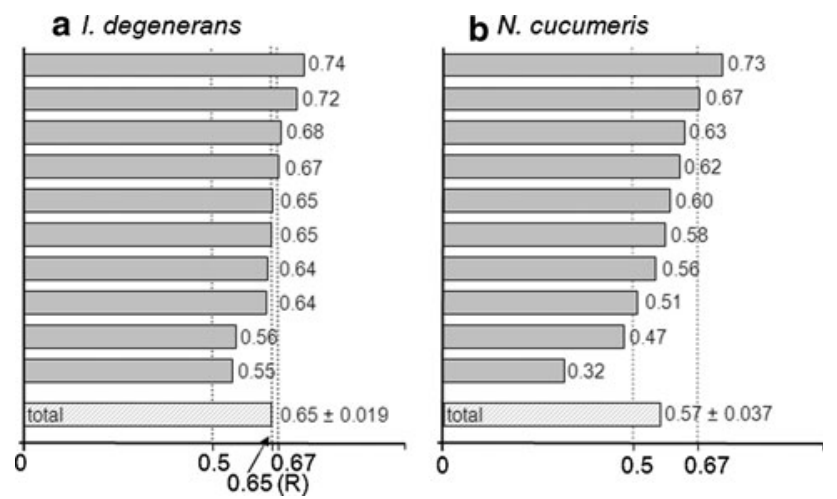

Fig. 4 The fraction of mites on the low-competition patches after $48 \mathrm{~h}$ for a I. degenerans and b N. cucumeris. Grey bars show the results for each individual replicate. The "total" bar shows the average of the ten replicates. Numbers next to the bars give the exact values of the fractions

distribution (Fig. 4a); neither did the average $(t=0.38$, $d f=9, p=0.72$.

Expected oviposition rates We expected equal oviposition rates of $I$. degenerans on the connected arenas (one and two holes with 75 mites), on the single patches with high competition (one hole) with 25 mites, and on the single patches with low competition (two holes) with 50 mites. Indeed, the number of eggs laid per mite did not differ between these treatments (Fig. 5a, ANOVA, $F=0.47, d f=2, p=0.63$ ), suggesting that the observed distribution of mites on the two connected patches resulted in an oviposition rate that did not differ from that expected.

\section{Neoseiulus cucumeris}

Movements of the mites Mites of N. cucumeris crossed the bridge between patches on average $13.2 \pm 0.57$ times in $48 \mathrm{~h}$, showing that the animals could easily move from one patch to the other.

Over the course of the experiment, the fraction of mites on the patch with low competition was higher than that on the patch with low competition, but the difference was not significant (LMER, $\chi^{2}=0.57, p=0.44$, Fig. $3 b$ ). The interaction of patch type with time was significant $\left(\chi^{2}=0.50, p=0.039\right)$, suggesting that the distribution of the mites over the patches changed during the experiment.

Distribution of the mites After $48 \mathrm{~h}$, on average, the fraction of the N. cucumeris mites found on the patch with low competition was 0.57 . This distribution differed significantly from a random distrubution in six out of ten replicates, and from the food accessibility rate $(0.67)$ in four out of ten (Fig. 4b). The heterogeneity among replicates was highly significant $\left(G_{\mathrm{H}}=62.9, \quad d f=9\right.$,
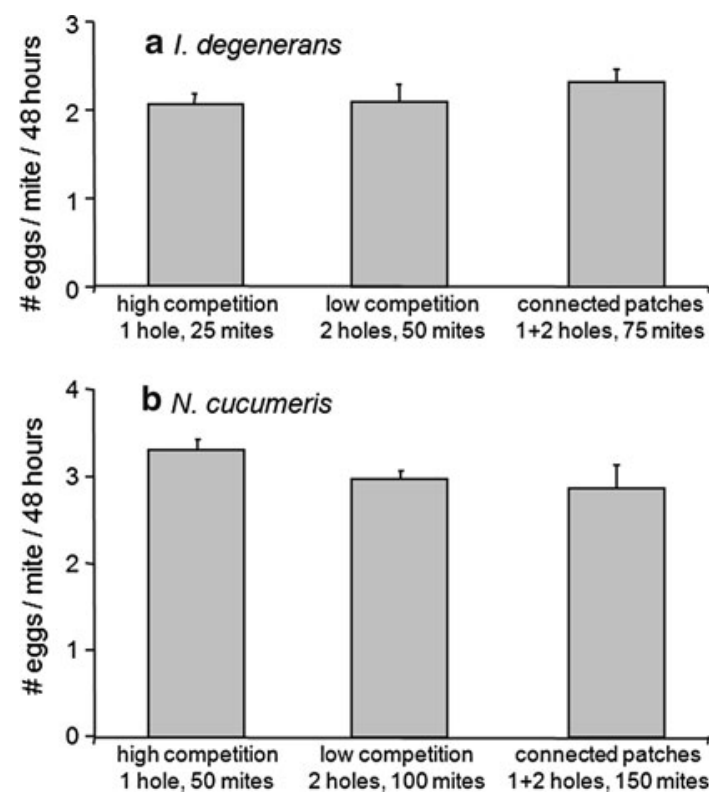

Fig. 5 Number of eggs produced per mite in $48 \mathrm{~h}$ for those treatments where the ratio between holes and number of mites was equal, hence indicating equal competition (25 per hole for $I$. degenerans and 50 per hole for $N$. cucumeris). The first two bars are from single patch experiments, and the third bar is from experiments with connected patches (IFD). a I. degenerans, b $N$. cucumeris

$p<0.001$ ), indicating high variation among replicates. The average distribution did not differ significantly from random $(t=1.88, d f=9, p=0.093)$, but differed from the food accessibility rate $(t=2.65, d f=9, p=0.026)$.

Because there was no interference for this species when there was unlimited access to the food (Fig. 2b), and we therefore combined data with similar ratios of numbers of mites per hole (see "Regression analysis" above), the expected distribution based on maximization of oviposition was the same as that based on food accessibility rates. We therefore conclude that $N$. cucumeris distributed themselves neither according to food accessibility rates nor according to the distribution predicted from maximization of oviposition.

Expected oviposition rates If they had distributed themselves according to the IFD, $N$. cucumeris was expected to experience the same level of competition on connected arenas as on single patches with high competition and 50 mites and on single patches with two holes and 100 mites. Although the distribution of $N$. cucumeris did not fit the expected distributions (see "Distribution of the mites" above), the number of eggs per mite did not differ between these treatments (ANOVA, $F=1.5, d f=2, p=0.24$, Fig. 5b), suggesting that the realized oviposition did not differ from that expected under an IFD. 


\section{Discussion}

The key prediction of the IFD theory is that animals are expected to distribute themselves such that no individual can increase its fitness by moving to another patch (Fretwell and Lucas 1969; Parker 1978; Milinski and Parker 1991). The IFD predicts habitat matching when the resource distribution is continuous and when there is no interference (Sutherland and Parker 1992; Tregenza 1995). However, the assumptions of the continuous input model are difficult to fulfil experimentally. We suggest that density-dependent estimates of fitness traits can be used to predict the distribution of animals instead. In this study, experiments on single patches were used to get such estimates, showing that the expected distribution based on oviposition rates for $I$. degenerans deviated slightly from food accessibility rates.

With ample food, experiments on single patches showed reduced oviposition by $I$. degenerans with increased densities of mites, suggesting the occurrence of interference in the absence of competition for access to food. We are unaware of the mechanism causing this negative regression, as we did not observe antagonistic interactions in the experiments. However, antagonistic behaviour of larger individuals directed toward smaller stages is common for $I$. degenerans. Indeed, smaller stages are highly vulnerable to cannibalism (Montserrat et al. 2006). Therefore, I. degenerans may have anticipated future cannibalism of the juveniles, and the high densities of I. degenerans may have caused some of the mites to attempt to disperse, thus allocating less time and energy to oviposition, causing a lower oviposition rate. Neoseiulus cucumeris adults also cannibalize on conspecific juveniles, but to a lesser extent.

Density effects as observed in this study are common (Gillis and Kramer 1987, Begon et al. 1990) and should therefore be taken into account when testing IFD theory. Here, the approach used to restrict the access to a food source may have intensified interference among competitors, because the mites were required to aggregate around the holes with food, which probably increased their encounter rates. However, in real systems, food will also often be highly patchily distributed. For example, groups of I. degenerans aggregate in the flowers on sweet pepper, where they feed on pollen (Faraji et al. 2002a).

The observed distribution of I. degenerans fitted well with the predicted distributions based on food accessibility and that based on oviposition. This mite may have perceived each hole as a separate patch, which would result in the same distribution as that based on food accessibility. Furthermore, the oviposition rate over $48 \mathrm{~h}$ equalled the expected oviposition based on the single patch experiments (Fig. 5a). In contrast, the observed distribution of females of the other predatory mite, $N$. cucumeris, undermatched the distribution predicted by food accessibility rates, even though six out of ten replicates actually fitted the distribution (Fig. 4b). Hence, the observed distribution also did not match that predicted when the mites perceived each hole as a separate patch. In addition, the overall distribution did not differ significantly from a random distribution, mainly due to large amounts of variation (Fig. 4b). However, there was a trend for more than $50 \%$ of the mites to move to the lowcompetition patches. It is possible that the prediction based on oviposition was caused by inaccurate estimates of the level of interference for $N$. cucumeris. Even though the regression fitted the data well (Fig. 2b), it is based on only a few densities. It is also likely that $N$. cucumeris needs more time to reach a stable distribution. Figure 3 demonstrates that the distribution of $N$. cucumeris shows high variation and may still be changing after $48 \mathrm{~h}$, so longer-lasting experiments are needed to exclude the possibility that $N$. cucumeris was still in the process of adjusting its distribution. Neoseiulus cucumeris also crossed the bridge half as often per mite than I. degenerans, suggesting lower mobility. Nevertheless, the oviposition rate of $N$. cucumeris did not differ from that expected based on the oviposition rates on single patches (Fig. 5b). Hence, although $N$. $\mathrm{cu}$ cumeris distributed themselves different from the IFD, this did not result in a change in oviposition rate, suggesting that oviposition rates of $N$. cucumeris may be (partially) independent of the distribution of animals. One reason for this deviation may be that a fraction of $N$. cucumeris might have spent substantial time on activities other than searching for food. This could occur if the searching time for the food was relatively short and the time between feeding activities was relatively long, resulting in a fraction of mites not experiencing competition. These mites would then distribute themselves independently of food accessibility, resulting in a range of distributions for which the mites have similar oviposition rates. Indeed, the variability among replicates in the distribution of $N$. cucumeris was much larger than among replicates of $I$. degenerans (Fig. 3). In addition, a much larger fraction of $N$. cucumeris was found away from the food sources, elsewhere on the patches, compared to I. degenerans (personal observation), suggesting that $N$. cucumeris were less inclined to search for food at any given time. Hence, differences between the two predatory mites may have been caused by differences in the level of competition for access to the food, which may have been stronger for I. degenerans. In addition, $N$. cucumeris may be better adapted to a pollen diet than I. degenerans. As models of the IFD do not consider fitness-determining behaviours other than foraging, it is essential to ensure that competition for food is strong and constant when testing this theory, such that the animals do indeed spend most of their time searching for food. This may not have been the case for N. cucumeris. 
In conclusion, we suggest that density-dependent fitness traits should always be assessed in studies on the IFD. They provide essential information about the strength and occurrence of interference, and about the expected fitness estimates, which enables the essential test of whether the distribution changes fitness traits. Interference may also vary with competitor density (Tregenza 1994; Van der Meer and Ens 1997; Moody and Houston 1995; Moody and Ruxton 1996). Aside from the animals not being ideal or free (Abrahams 1986, Fretwell 1972; Holmgren 1995; Hugie and Grand 1998), this could be another reason that competitors are not distributed according to the difference in food availability in experiments.

Acknowledgments Martijn Egas, Nicola Tiën, Roos van Maanen, and Paulien de Bruijn are thanked for valuable discussions. TvdH and MM received an ALW grant (TvdH no. 813.04.011, MM no. 814.01.006) from the Netherlands Organization for Scientific Research (NWO).

Open Access This article is distributed under the terms of the Creative Commons Attribution Noncommercial License which permits any noncommercial use, distribution, and reproduction in any medium, provided the original author(s) and source are credited.

\section{References}

Abrahams MV (1986) Patch choice under perceptual constraints-a cause for departures from an ideal free distribution. Behav Ecol Sociobiol 19:409-415

Begon M, Harper JL, Townsend CR (1990) Ecology: individuals, populations and communities. Blackwell, Oxford

Charnov EL (1976) Optimal foraging, the marginal value theorem. Theor Popul Biol 9:129-136

Choh Y, Van der Hammen T, Sabelis MW, Janssen A (2010) Cues of intraguild predators affect the distribution of intraguild prey. Oecologia 163:335-340

De Moraes GJ, McMurtry JA et al (2004) A revised catalog of the mite family Phytoseiidae. Zootaxa 434:1-494

Denmark HA, Edland T (2002) The subfamily Amblyseiinae Muma (Acari: Phytoseiidae) in Norway. Int J Acarol 28:195-220

Earn DJD, Johnstone RA (1997) A systematic error in tests of ideal free theory. Proc R Soc Lond Ser B 264:1671-1675

Faraji F, Janssen A, Van Rijn PCJ, Sabelis MW (2000) Kin recognition by the predatory mite Iphiseius degenerans: discrimination among own, conspecific, and heterospecific eggs. Ecol Entomol 25:147-155

Faraji F, Janssen A, Sabelis MW (2002a) Oviposition patterns in a predatory mite reduce the risk of egg predation caused by prey. Ecol Entomol 27:660-664

Faraji F, Janssen A, Sabelis MW (2002b) The benefits of clustering eggs: the role of egg predation and larval cannibalism in a predatory mite. Oecologia 131:20-26

Flaxman SM, deRoos CA (2007) Different modes of resource variation provide a critical test of ideal free distribution models. Behav Ecol Sociobiol 61:877-886

Fretwell SD (1972) Populations in a seasonal environment. Princeton University Press, Princeton

Fretwell SD, Lucas HL (1969) On territorial behavior and other factors influencing habitat distribution in birds. Acta Biotheor $19: 16-36$
Gillis DM, Kramer DL (1987) Ideal interference distributionspopulation-density and patch use by zebrafish. Anim Behav 35:1875-1882

Hassell MP, Varley GC (1969) New inductive population model for insect parasites and its bearing on biological control. Nature 223:1133-1137

Haugen TO, Winfield IJ, Vollestad LA, Fletcher JM, James JB, Stenseth NC (2006) The ideal free pike: 50 years of fitnessmaximizing dispersal in Windermere. Proc R Soc B Biol Sci 273:2917-2924

Holmgren N (1995) The ideal free distribution of unequal competitors-predictions from a behavior-based functional response. J Anim Ecol 64:197-212

Hugie DM, Grand TC (1998) Movement between patches, unequal competitors and the ideal free distribution. Evol Ecol 12:1-19

Kacelnik A, Krebs JR, Bernstein C (1992) The ideal free distribution and predator-prey populations. Trends Ecol Evol 7:50-55

Kennedy M, Gray RD (1993) Can ecological theory predict the distribution of foraging animals-a critical analysis of experiments on the ideal free distribution. Oikos 68:158-166

Milinski M (1984) Competitive resource sharing-an experimental test of a learning rule for ESSs. Anim Behav 32:233-242

Milinski M, Parker GA (1991) Competition for resources. In: Krebs JR, Davies NB (eds) Behavioural ecology: an evolutionary approach, 3rd edn. Blackwell, Oxford, pp 137-168

Montserrat M, Janssen A, Magalhaes S, Sabelis MW (2006) To be an intraguild predator or a cannibal: is prey quality decisive? Ecol Entomol 31:430-436

Moody AL, Houston AI (1995) Interference and the ideal free distribution. Anim Behav 49:1065-1072

Moody AL, Ruxton GD (1996) The intensity of interference varies with feed density: support for behaviour-based models of interference. Oecologia 108:446-449

Morris DW (1989) Density-dependent habitat selection-testing the theory with fitness data. Evol Ecol 3:80-94

Parker GA (1978) Searching for mates. In: Krebs JR, Davies NB (eds) Behavioural ecology: an evolutionary approach, vol 1 . Sinauer Associates, Sunderland, pp 214-244

Parker GA, Sutherland WJ (1986) Ideal free distributions; when individuals differ in competitive ability-phenotype-limited ideal free models. Anim Behav 34:1222-1242

Pulliam HR, Caraco T (1984) Living in groups: is there an optimal group size? In: Krebs JR, Davies NB (eds) Behavioural ecology: an evolutionary approach, vol 2. Blackwell, Oxford, pp 122-147

R Development Core Team (2008) R: A language and environment for statistical computing. R Foundation for Statistical Computing, Vienna. http://www.R-project.org

Sokal RR, Rohlf FJ (1995) Biometry, 3rd edn. WH Freeman, New York Sutherland WJ (1983) Aggregation and the ideal free distribution. J Anim Ecol 52:821-828

Sutherland WJ, Parker GA (1985) Distribution of unequal competitors. In: Sibly RM, Smith RH (eds) Behavioural ecology: ecological consequences of adaptive behaviour. Blackwell, Oxford, pp 255-274

Sutherland WJ, Parker GA (1992) The relationship between continuous input and interference models of ideal free distributions with unequal competitors. Anim Behav 44:345-355

Tregenza T (1994) Common misconceptions in applying the ideal free distribution. Anim Behav 47:485-487

Tregenza T (1995) Building on the ideal free distribution. Adv Ecol Res 26:253-307

Tregenza T, Parker GA, Thompson DJ (1996a) Interference and the ideal free distribution: models and tests. Behav Ecol 7:379-386

Tregenza T, Thompson DJ, Parker GA (1996b) Interference and the ideal free distribution: oviposition in a parasitoid wasp. Behav Ecol 7:387-394 
Van Rijn PCJ, Tanigoshi LK (1999) Pollen as food for the predatory mites Iphiseius degenerans and Neoseiulus cucumeris (Acari : Phytoseiidae): dietary range and life history. Exp Appl Acarol 23:785-802

Van der Meer J, Ens BJ (1997) Models of interference and their consequences for the spatial distribution of ideal and free predators. J Anim Ecol 66(6):846-858
Vantornhout I (2006) Biology and ecology of the predatory mite Iphiseius degenerans (Berlese) (Acari: Phytoseiidae) (Ph.D. thesis). Ghent University, Ghent

Yao DS, Chant DA (1990) Changes in body weight of two species of predatory mites (Acarina: Phytoseiidae) as a consequence of feeding in an interactive system. Exp Appl Acarol $8: 195-220$ 